Maija Nousiainen (MSc) works in the Department of Physics, University of Helsinki, in The Finnish Graduate School of

Mathematics, Physics and Chemistry. Her research activity focuses on the use and development of concept maps in physics

teacher education. In addition to research, she has acted as an instructor in physics teacher courses since year 2006.

Ismo T. Koponen (PhD) works as a university lecturer in the Department of Physics, University of Helsinki. His research

activities concentrate on Physics Education Research, but include also computational and statistical physics. Since 1999 he

has taught advanced courses pre-service physics teachers in the Physics Department. In addition, he acts as a supervisor of

$\mathrm{MSc}$ and $\mathrm{PhD}$ thesis done in physics education.

MAIJA NOUSIAINEN* and ISMO T. KOPONEN

Department of Physics, University of Helsinki

maija.nousiainen@helsinki.fi

ismo.koponen@helsinki.fi

*) née Pehkonen

\title{
Concept maps representing knowledge of physics: Connecting structure and content in the context of electricity and magnetism
}

\begin{abstract}
Many assume that the quality of students' content knowledge can be connected to certain structural characteristics of concept maps, such as the clustering of concepts around other concepts, cyclical paths between concepts and the hierarchical ordering of concepts. In order to study this relationship, we examine concept maps in electricity and magnetism drawn by physics teacher students and their instructors. The structural analysis of the maps is based on the operationalisation of important structural features (i.e. the features of interest are recognised and made measurable). A quantitative analysis of 43 concept maps was carried out on this basis. The results show that structure and content are closely connected; the structural features of clustering, cyclicity and hierarchy can serve as quantitative measures in characterising structural quality as well as the quality of content knowledge in concept maps. These findings have educational implications in regard to fostering the teacher student's organisation of knowledge and in monitoring the process of such organisation.
\end{abstract}

\section{INTRODUCTION}

In teaching and learning as well as in educational research it is widely assume that graphical knowledge representation tools such as concept maps help students to organise their knowledge around the most important concepts and principles of the subject content (Novak \& Gowin 1984; Ruiz-Primo \& Shavelson, 1996; Ingeç, 2008; Nesbit \& Adesope, 2006). Recent research focusing on the structure of the concept map suggests that a good understanding and the high quality of students' knowledge are reflected as interconnected and web-like structures (Vanides, Yin, Tomita \& Ruiz-Primo, 2005; Ingeç, 2008; van Zele, Lenaerts \& Wieme, 2004; Kinchin, Hay \& Adams, 2000; Kinchin, De-Leij \& Hay, 2005; Safayeni, Derbentseva \& Cañas, 2005; Derbentseva, Safayeni \& Cañas, 2007). The notion that structural features and the quality of students' understanding may be connected warrants a closer examination of the structure of such maps. Furthermore, this notion also warrants an attempt to render the interesting structural features measurable.

Several studies have pointed out that in certain disciplines such as physics and biology the concept maps tend to be hierarchical - possibly reflecting a hierarchical ordering of concepts - whereas in other areas (e.g. chemistry) non-hierarchical maps are expected because the underlying structure 
of knowledge is not necessarily hierarchical (Novak \& Gowin, 1984; Zoller, 1990; Ruiz-Primo \& Shavelson, 1996; van Zele et al., 2004). Moreover, other studies have suggested that topological features suh as chains, spokes and nets carry important information about the quality of knowledge represented in such maps not easily captured by straightforward quantitative methods of analysis. (Vanides et al., 2005; Kinchin et al., 2000, 2005; Ingeç, 2008). However, this study aims to show that the topological features of web-like structures are equally amenable to quantitative description.

Interconnectedness and cyclicity are characteristics of ways representing and arranging knowledge which allow the learner to proceed through paths in the conceptual space where learning takes place (Kinchin et al., 2000, 2005). Most of the research on concept maps views hierarchy and web-like connectedness as somehow separate (or even contradictory) features. This can be interpreted as a question of the design principles of the concept maps. If the design principles restrict the potential to express complex knowledge (e.g. a strict rule to form propositional nodelink-node connections as in traditional concept maps or mind maps), this will also narrow the cognitive space of learning. However, hierarchical organisation and interconnectedness need not be contradictory properties. Rather, they may well be coexistent, mutually supporting features.

A standard method to evaluate concept maps is to compare them to a "master map" (i.e. a map constructed by experts in the subject content, see Ruiz-Primo and Shavelson, 1996). In order to visualise the relevant topological features, it is useful to make the visual appearance of the maps comparable by removing the ambiguity associated with personal styles of graphical layout. This can be carried out by redrawing the maps so that the same rules for ordering the nodes are used in all cases. The maps are redrawns with COMBINATORICA software (Pemmaraju \& Skiena, 2006) and contain the same information as the original ones (i.e. the original and the redrawn maps are isomorphic representations of the same node-link-node systems).

In this study, we develop a method of analysis which captures the important qualitative features of concept maps: the hierarchical ordering of concepts (how one concept is sub-ordinate to other concepts), the clustering of concepts around other concepts (local interconnectedness) and cyclical paths between concepts (global interconnectedness). The concept maps analysed here were originally designed for purposes of representing knowledge structures in physics (electricity and magnetism) and were produced in an advanced-level the physics teacher education (third and fourth year of studies). The concept maps were constructed by following design principles where concepts here include also quantities and laws, and where links represent procedures which include either certain types of quantitative experiments in physics. In this study we further develop the theoretical method of analysis and apply the method to a more extensive sample of concept maps than in our previous study (Koponen \& Pehkonen, 2010).

\section{DESIGN PRINCIPLES OF CONCEPT MAPS}

In most research, questions of structure are limited to concepts maps, where connections are simple propositions (Novak \& Gowin, 1984; Ruiz-Primo \& Shavelson, 1996; McClure, Sonak \& Suen, 1999) and are thus limited only to a certain type of propositional knowledge. However, generalised rules are needed in order to represent more complex relations and relational structures. Previously, we introduced design principles which rest on the use of quantitative experiments and models (Koponen \& Pehkonen, 2010; Pehkonen, Koponen \& Mäntylä, 2009; Koponen \& Mäntylä, 2006).

In the quantitative experiment, the concept is operationalised (i.e. rendered measurable through pre-existing concepts). For example, the operationalisation of Coulombs' law requires the concept of force and charge, whereas the concept of the electrical field rests on force, charge and Coulomb's law, and so on. This mutual dependence of concepts means that a network of concepts 
is woven through operationalisation. Although many different types of experiments are relevant to teaching and learning, quantitative experiments play a special role because they can serve to support the construction of new concepts and new relations between concepts (laws) on the basis of concepts already known ones (Koponen \& Mäntylä, 2006). In such experiments, a new concept or law is always constructed sequentially, starting from those that already exist and which also provide the basis for an experiment's design and interpretation.

The results of the experiments and how they modify knowledge structures are expressed and represented in terms of models. Therefore, in addition to experiments, models are also core components of knowledge structures, as well as of knowledge itself. For example, the definition of the electrical field can be seen as a model which breaks the force between two interacting charges into one part which causes the field (the charge as a source) and another part which experiences the field (the other charge). Another example is the model of a homogeneous field, extensively used as a model in introductory electricity. Typically, a model may be an idealised and symbolic representation or a description of dependencies found in an experiment or that should provide explanations and predictions of regularities found in experimental data (Koponen, 2007; Sensevy, Tiberghien, Santini, Laube, \& Griggs, 2008).

Consequently, the design of concept maps discussed here is based on a special type of selection of nodes (concepts) and special types of links connecting the nodes. The nodes can be:

1. Concepts or quantities.

2. Laws (particular or general).

Of these elements, laws could be taken as particular experimental laws or law-like predictions in specific situations (derived from a theory). General laws are more fundamental principles (e.g. principles of conservation). In both cases, laws can be expressed as relations between concepts. The links are thus:

3. Experimental procedures (an operational definition).

4. Modelling procedures, which can be deductive models or definitions in terms of a modeltype relations.

These procedures play a central role in providing order and organisation to the whole conceptual structure. The basic idea is that the design principles guide the construction process of the map. It should be noted that students must ensure that every link they draw on the map is a procedure (either experimental or modelling) and justify them separately in a written report. Finding physically unacceptable connections in the students' concept maps is therefore is unlikely: rather, their maps would contain only connections with a variable degree of justification as the poor quality reflects lack of connections and vagueness in justifying them.

\section{THE RESEARCH SETUP AND THE RESEARCH QUUESTIONS}

The design principles explicated as elements 1-2 and rules 3-4 served to construct concept maps during physics teacher education courses over three academic years from 2006 to 2009. The courses were similar; each was of seven weeks' duration and focused on questions concerning the conceptual structure of physics. Here we discuss the structure of concept maps (the total number of student and instructor maps was 43) made during the course in the context of electricity and magnetism. During the teaching sequence, the students produced an initial concept map, and later, after instruction and group discussions, final maps (the total number of student maps was 39). The course instructors collaboratively produced four concept maps to plan the teaching, the content of the maps closely followed the content of the standard introductory course on electromagnetism. Of these instructor-made maps, one small map with $n=17$ concepts (i.e. nodes) and three large maps with $n=34$ concepts were constantly updated so that they contained all physically wellmotivated and correct connections found in the student made concept maps throughout the cour- 
ses.. In this article, these maps will be called "master maps". In constructing the maps, the choice of concepts was restricted to a given set of elements, chosen to be either $n=17$ (15 student maps and 1 master map) or $n=34$ ( 24 student maps and 3 master maps), but the number of procedures was unrestricted, provided they were types 3 or 4 . The visual outlook of the maps appears later, in Figures 2 and 3.

It should be noted that students produced the maps in a rather advanced stage of their studies. The students were familiar with basic physics and the basic concepts. Concept mapping during the teaching sequence therefore served as a learning tool to organise the content already known and to transform previous knowledge into a more functional form. Additional details about concept map construction and course practices as well as collaboration between students is discussed in more detail elsewhere (Koponen \& Pehkonen, 2010; Pehkonen et al., 2009).

The basic question is how the structure of the concept map can be related to the content of the knowledge the map is intended to represent. In order to answer the basic question, the qualitative features must be made measurable, and suitable variables must be defined. In this article, the variables that measure the structural features are called structural variables. Thus, the research questions posed here are as follows:

1. Which structural variables can serve to indicate important qualitative features?

2. How are structural variables connected to the content of the maps?

3. How can we use structural variables to classify concept maps and their quality?

Answering the first question requires reducing the qualitative notions of being web-like and interconnected to properties of clustering, cyclicity and hierarchy, which can then be measured and ultimately operationalised in the form of six structural variables. Such structural analysis reveals how concepts are connected. It is worth remembering that all links represented in the maps represent "correct" knowledge in the sense that students have been able to justify the connections in terms of procedures. Therefore, the question of quality concerns the number and organisation of these connections, so there is no need to address the content, which for all practical purposes can be presumed as "correct". The answer to the second question is obtained through a detailed examination of the structure of the master map. It is shown that the chosen variables are capable of discerning concepts relevant to the content. The third question is answered by comparing the master map and students' maps on the basis of structural variables. The master map and student map is often compared by verifying which nodes and links are similar and ignoring those in agreement with the master map. This kind of comparison focuses on the exact similarities in the maps. In this study, instead of requiring exact similarity, we base the comparison of the structural similarities on the six variables that characterise the structure.

\section{THE METHOD OF ANALYSIS OF CONCEPT MAPS}

The purpose of the method of structural analysis is to operationalise the relevant structural features of hierarchy, clustering and cyclicity. In this context, hierarchy means the property of branching, where a set of concepts can be reached equally easily from a given concept. Clustering here means a property where concepts tend to form an interconnected cluster, and cyclicity refers to a property found when a set of concepts are connected by a closed path. In what follows, one should remember that all nodes represent concept-like entities, and links represent procedures. This is an important difference from a traditional concept map presented by, for example, Novak \& Gowin (1984), where linking words are verbs, and maps consist thus of concept-link-concept propositions.

The basic variables, indicate connections between nodes $i$ and $j$, are denoted by binary values $a_{i j}$ such that if nodes are connected $a_{i j}=1$ and in the absence of a connection, $a_{i j}=0$. In a map of $n$ nodes the variables $a_{i j}$ form a $n \times n$ dimensional adjacency matrix $\boldsymbol{a}$. The steps in analysing the structure are thus: 
1. Coding the connections in the map to a connectivity (adjacency) matrix.

2. Visual inspection using two different embeddings for each map.

3. Calculating the values for variables characterising the topology of the map.

4. Comparison of the structure and content of the maps.

Information about the directedness of the map connections is not taken into account, however, because in this study we focus only on the primary topological features.

\section{Visual inspection: The embeddings}

In order to visualise the relevant topological features, it is useful to make the visual appearance of the maps comparable by removing any ambiguity associated with the graphical layout. This can be done by redrawing the maps so that the same rules for ordering the nodes are used in all cases. In graph theory this is called embedding the graph, and for the embeddings several well-defined methods are available. The embeddings used in the present study were carried out using COMBINATORICA software (Pemmaraju \& Skiena, 2006). The embedded maps included the same information as did the originals (i.e. they were isomorphic representations).

We used two different graph-embedding methods, both of which are standard visualisation methods used for network data (see e.g Pemmaraju \& Skiena, 2006; Kolaczyk, 2009). The first method was spring-embedding, which is obtained when each link is presumed to behave like a "spring" (i.e. the linear restoring force when distance increases) and then minimising the total energy of the spring system. The energy is minimised iteratively until a stable structure (i.e. minimum energy of tension) is achieved (for details, see Pemmaraju \& Skiena, 2006). The methods for representing the network as a "spring" network were chosen because linear forces are easy to handle, and simple iterative schemes of energy minimisation are available (Kolaczyk, 2009). Spring-embedding serves the purpose of revealing visually how tightly certain concepts are connected, so it is suitable for visual inspection of the clustering and cyclical patterns. The second form of embedding was tree-embedding (sometimes also called root-embedding). In tree-embedding, the maps are redrawn as an ordered hierarchical tree with a certain node selected as a root. The nodes and links are then rearranged so that the nodes, which are equidistant (i.e. the same number of steps is needed to reach each node) from the root, are on the same hierarchical level. The hierarchical levels thus contain all those nodes which can be reached with the same number of steps from the root node. Tree embedding is therefore suitable for inspection of the hierarchical organisation of nodes.

\section{Definition of the variables}

Having recognised hierarchy, clustering and cyclicity as important features of concept maps we must find ways to operationalise these properties. The structural variables of importance (the subscript indicates that the measure is locally for a node $k$ ) are defined such that they correspond precisely to the topological features of clustering, cyclicity and hierarchy. Schematic examples of basic patterns such as clustering, cyclical and hierarchical appear in Figure 1.
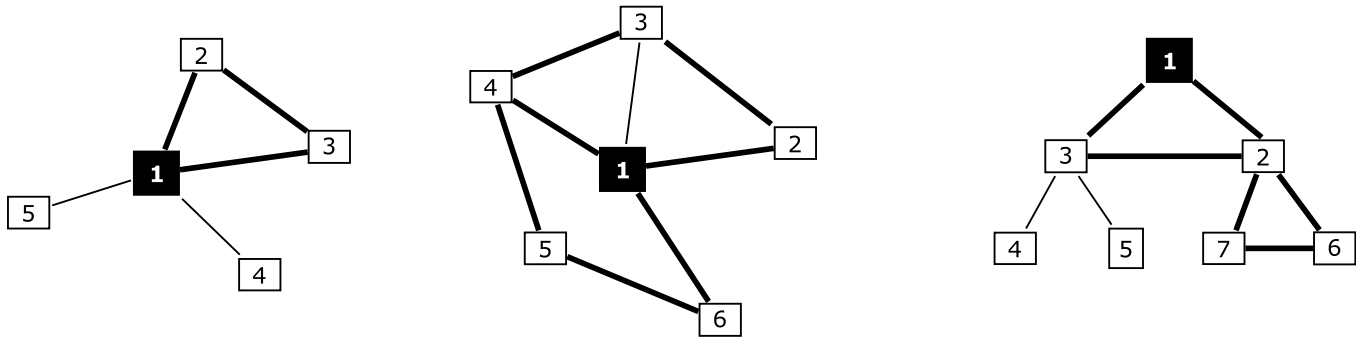

Figure 1. Clustering, cyclicity and hierarchy (from left to right) illustrated. The core concept is emphasised as a black square, and the structural features are viewed locally from the point of view of the core concept. 
The structural variables suitable for measuring the basic topological features of clustering, cyclicity and hierarchy can be defined as follows (mathematical definitions appear in Table I).

1. The degree of nodes $D_{k}$ is the number of links connected to a given node $k$. The degree $D_{k}$ contains the incoming and outgoing links.

2. The clustering coefficient $C_{k}$ measures the relative number of triangles of all triply connected neighbours around a given concept. $C_{k}$ obtains values between 0 and 1 , where 1 corresponds to the maximum number (depending on the number of neighbours) of triangles (all triply connected neighbours form triangles).

3. The subgraph centrality $S C_{k}$ measures the cyclicity (i.e. the number of subgraphs that constitute closed paths traversing through a node). A large value means that from the given node many other nodes can be reached through closed paths.

4. The transit efficiency $T_{k}$ measures the relative ease of passing through a given node. There are always several paths leading from node $i$ to node $j$ such that the path passes through node $k$, but some paths are shorter than others.

5. Hierarchy $H_{k}$, which measures the degree of hierarchy, is calculated as the sum of all hierarchy levels, but is weighted by a number of connections within a given level. Hierarchical levels are obtained from tree-embedding, which contains information on the path lengths $d_{i j}$ between nodes $i$ and $j$ (i.e. the number of steps needed to pass from $i$ to $j$ ). For perfect tree-like hierarchies with no intralevel connections, $H_{k}=0$; for fully connected structures, $H_{k}=1$. For hierarchies with a tree-like backbone and a number of intralevel connections (typical of structures with cycles) hierarchy will always be $H_{k}>1$.

The mathematical definitions of structural variables 1-3 are standard and are explained in greater detail in Costa, Rodrigues, Travieso and Villas Boas (2007) and Kolczyk (2009). The definition of variable 4 is a slightly modified version of harmonic distance (Costa et al., 2007; Kolaczyk, 2009), whereas the hierarchy is defined according to McClure et al. 1999. All of variables 1-5 can be expressed in detailed mathematical definitions, which are summarised in Table I and can be calculated when the variables for $a_{i j}$ (adjacency matrix a) are known.

Table I. Mathematical definitions of variables for measuring the topology of the concept maps. The subscripts $k$ refer to kth node, the number of nodes is $N$, and the number of links $M$. The adjacency matrix is given by $\boldsymbol{a}$ and has elements $a_{i j}$. The matrix of the shortest paths is $\boldsymbol{d}$ (elements $d_{i j}$ ) and is obtained from hierarchical tree-embeddings. The tree-embedding, which begins from node $k$, also yields the number of hierarchical levels $l_{k}$ with $n_{k}(l)$ cross links.

\begin{tabular}{lll}
\hline Variable & \multicolumn{2}{l}{ Definition } \\
Degree of node & $D_{k}$ & $\sum_{i}\left(a_{i k}+a_{k i}\right)$ \\
Clustering & $C_{k}$ & $\sum_{i>j} a_{k j} a_{i j} a_{j k} / \sum_{i>j} a_{i k} a_{k j}$ \\
Subgraph centrality & $S C_{k}$ & $\left(\sum_{i}\left(\boldsymbol{a}^{i}\right)_{k k} / i !\right) /\left(\sum_{i} D_{i} / N\right)$ \\
Transit efficiency & $T_{k}$ & $\left(\sum_{i, j}\left(d_{i k}+d_{k j}\right) / d_{i j}\right)^{-1}$ \\
Hierarchy & $H_{k}$ & $\sum_{l} l_{k} n_{k}(l) /(N-1)$ \\
Importance & $I_{k}$ & $C_{k} \times S C_{k} \times T_{k}$ \\
\hline
\end{tabular}


The variables in Table I provide information on the different but closely related structural aspects of the concept map and are therefore first calculated for each node in the map. This study will show that the coefficients $C_{k}, S C_{k}$ and $T_{k}$ more or less correlate because they measure different aspects of the centrality of the node (concept) in the whole structure. This suggests that we can reduce the information by requiring that the node, which is structurally important and clusters other nodes around it, have a high value for all observables $C_{k}, S C_{k}$, and $T_{k}$ and thus define the importance of clustering and cyclicity, or simply the importance $I_{k}$, of the node as a product

$$
I_{k}=C_{k} \times S C_{k} \times T_{k}
$$

This variable has a high value if the node gathers other nodes around it and if at the same time many other nodes are easily accessed through it. Therefore, in the analysis, the variables $C_{k}, S C_{k}$ and $T_{k}$ are used only in the calculating of the compound variable $I_{k}$ which has a high value when all variables measuring clustering and cyclicity have high values (high local and global interconnectedness), but a low value when even one of the three variables measuring clustering has a value close to zero. The final evaluation and comparison of the structure are carried out on the basis of $D_{k}, H_{k}$ and $I_{k}$.

\section{Comparison of the concept maps: Connecting the structure and content}

In order to compare the students' concept maps with the above studied $n=17$ and $n=34$ master maps (denoted by MM), we needed to reduce the total information. In order to do that we concentrated only on $I_{k}$ and $H_{k}$, and compared their values in the students' maps to those in the master map by taking a "projection" of the variables. This is carried out by representing the variables as vectors $\boldsymbol{X}$ and calculating the projected value $X_{P}$ of the variables as a scalar-product

$$
X_{P}=(1 / L)\left(\boldsymbol{X}_{M M} \cdot \boldsymbol{X}\right)^{1 / 2} ; \quad L=\left(\boldsymbol{X}_{M M} \cdot \boldsymbol{X}_{M M}\right)^{1 / 2}
$$

where $L$ is the normalisation factor chosen such that the projected values (length of the vectors) are the same for the original maps as for the master maps. The purpose of the comparison is to determine whether the concepts and laws in student maps have a similar structural position to that of the master maps. This study will later show that different concepts in the master maps then fall into different classes, making it clear that the hierarchy $H_{k}$ and importance $I_{k}$ are directly related to the content relevance of the concepts. Values of $X_{P}$ close to 1 now require that the value sets of the variables be close to each other in both the students' concept maps and the master maps ( i.e. the same concepts are in similar ways structurally important). A value of 0 means that no structural similarities exist, or alternatively that structurally important concepts differ entirely from the master maps. Comparison on the basis of the projection has the advantage that in it we first define the structural properties of interest $(H$ and $I)$ and then compare them on the basis of the corresponding structural variables. Then the structural analysis and the comparison both rest on the same theoretical footing, which allows us to couple both the content and structure. This finally motivates us to define the quality of the map as the product $H_{P} \times I_{P}$. The justification for this type of comparison is discussed in greater detail in the next section.

\section{RESULTS}

The sample of concept maps studied here consists of $\mathrm{N}=43$ maps. Of these maps, 4 were produced collaboratively by physics teacher instructors (physicists) and 39 were produced by physics student teachers. Of the 43 maps, 14 have $n=17$ concepts and laws and 29 have $n=34$ nodes (concepts and laws). The largest number of links appears the master maps, with 28 links in the $n$ $=17$ map, and 69 links in the $n=34$ map. All these maps are studied as one sample, because the properties of interest depend not on $n$, but rather on $D$. 


\section{Examples of the concept maps}

The master maps with $n=17$ concepts appears in Figure 2, and an example of a $n=34$ map is in Figure 3. These are the extensive maps contained in the sample, because they are constructed such that they contain all relevant connections (i.e. evaluated by the instructors as physically correct and logically sound) also found in the students' concept maps. Several student map, also share a similar appearance and come close to the examples shown in Figures 2 and 3. In these kinds of maps, the most well connected concepts are typically linked to 3-5 other concepts. As explained before, all the connections shown in the map are experimental or modelling procedures formed on the basis of rules 3 and 4 . Some of the most important nodes and connecting procedures appear in Table II with numbering that refers to Figures 2 and 3. It is worth noting that the numbering of links denotes the sequence of steps in the construction of the concept maps.

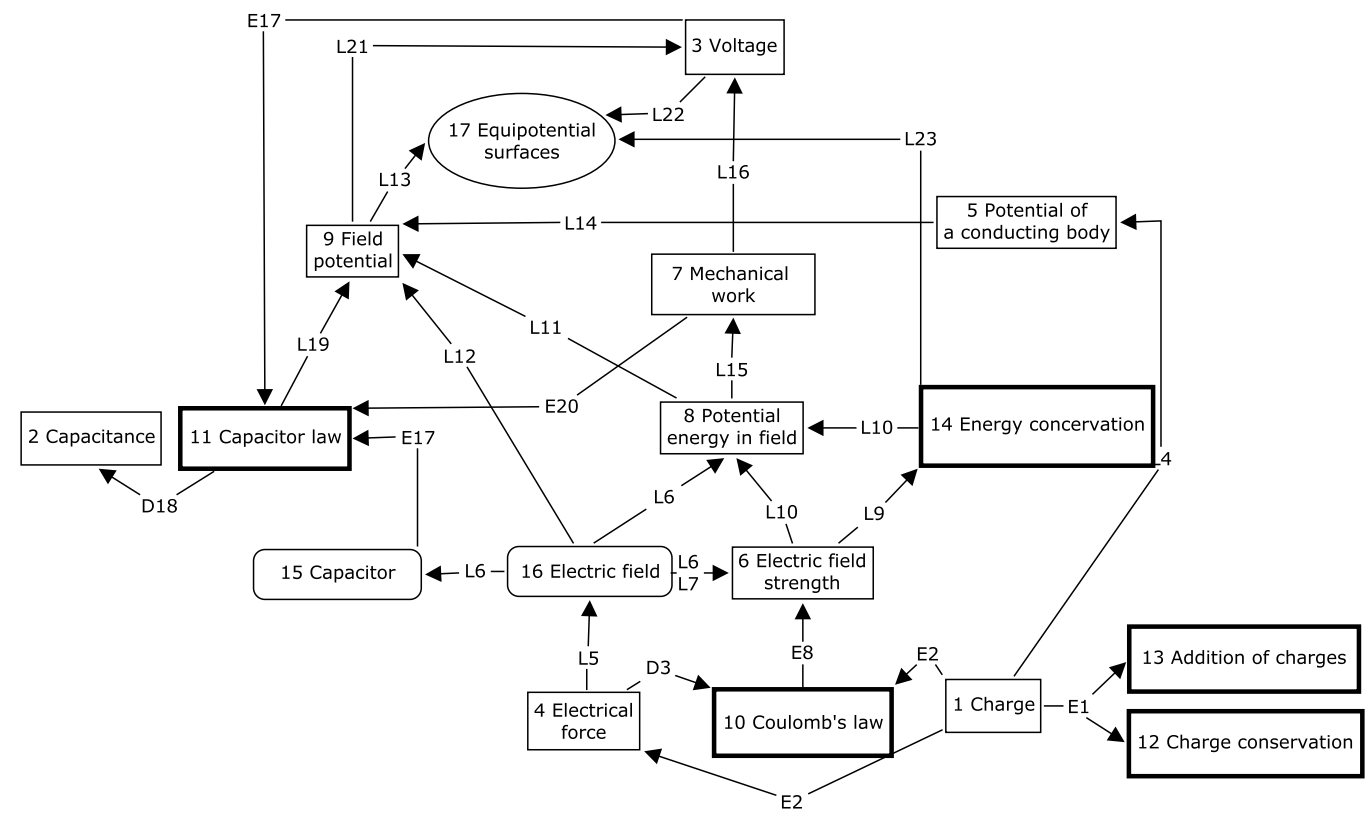

Figure 2. The master map for $n=17$ concepts in electrostatics. The map shows concepts (boxes), laws and principles (boxes with thick borders). Links are either operationalising experiments (E) or modelling procedures (either definition is denoted by $D$ or by logical deductions $L$ ). Experimental setups (model) appear shown as elliptical, entity-like objects with rounded boxes.

The triangular and tree-like patterns are now visible in several places in the concept maps in Figures 2 and 3 . These simple patterns are actually quite central to the maps, as is the content contained in these patterns. In what follows, we discuss in some detail the patterns related Coulomb's law and the electrical field.

Coulomb's law. In map $n=17$ the triangular pattern leading to Coulomb's law (10) requires qualitative notions of a charge (1) and repulsive/attractive electrical forces (2) resulting from a charge (or charging). Then a particular idealised experiment, Coulomb's experiment (E2) with spherical capacitors, can be designed. The outcome of this experiment is the symbolic form of Coulomb's law. This finally enables one to measure of the charges and defines the quantity of the charge through Coulomb's law. Experiment E17 leading to the capacitor law and the quantification of 


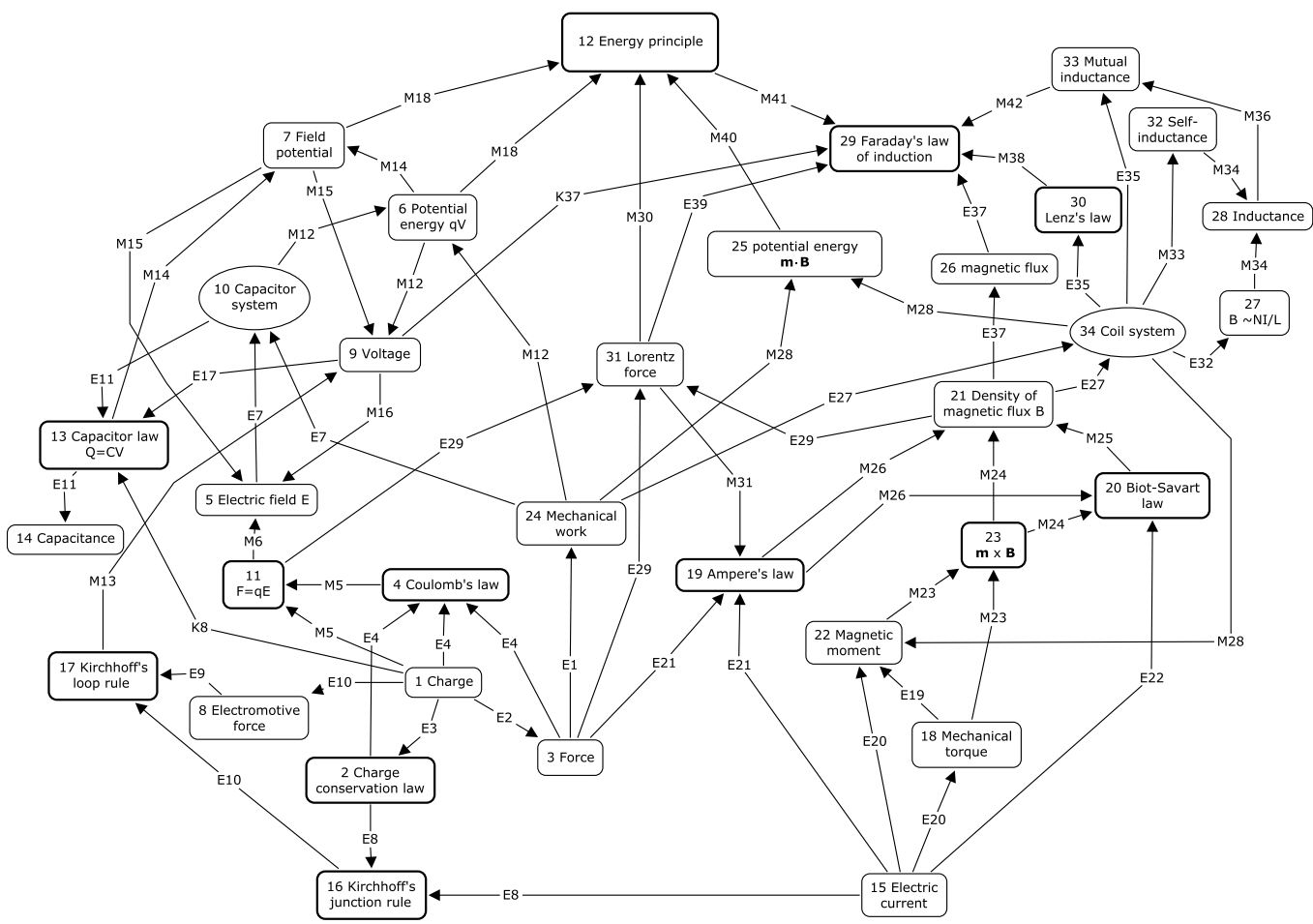

Figure 3. The master map for $n=34$ concepts in electromagnetism. Symbols appear as in Figure 2.

capacitance is very similar in structure. Some experiments, such as E8 and E20, are considerably simpler, because in them only one aspect in the situation is changed. In E8, the test charge (small vs. large charge) is introduced, and its position is changed, thereby enabling one to define of electrical field strength. In E20, the test charge is displaced in the field between the plates of a planar capacitor system, enabling one to generalise of mechanical work for electrical fields.

The electrical field. Most of the (deductive) modelling examples are designed to explain and clarify certain experimental situations and are often already included as part of the experimental procedures. For example, in the $n=17$ case shown in Figure 2, E2 is the model of radial force lines and point charges; E17 introduces the homogeneous field analogous to a gravitational field. Another type of modelling is used when the electrical field (16) is first introduced in L6 as a concept redundant to electrical force (the force is divided by the smaller charge), but is then idealised and generalised so that it is understood and defined (L7) as an entity created by a charge and which is felt by another charge. This definition may use Coulomb's law, which in turn enforces the position of Coulomb's law as a basic law of electrostatics. The concept of field (16 and 6) may be based on the notion of the potential energy of electrical interaction by using an analogous model with a gravitational field (L10). This completes a triangular cycle consisting of nodes 16, 6 and 8, and links L6, L7 and L10. The analogous model with a gravitational field is also used in the triangle consisting of nodes 6,8 and 10, where the principle of energy conservation is applied to an electrical field (L10). When these structures are viewed a bit differently, we can recognise the hierarchical deductive chains (e.g. one beginning from 16 and continuing to 8 and then from 8 to 7 and 9) through links L6, L15 and L11, where. L15 generalises the idea of mechanical work for the electrical field by using a line integral representation. 
Table II: Examples of concepts and procedures in the instructors' concept maps. The larger map with $n=34$ nodes contains nearly all the concepts contained in the smaller map with $n=17$. The numbering is given in the order $(n=17 / n=34)$. In the map with $n=34$ includes altogether 69 procedures, only the most important of which appear in the table.

\begin{tabular}{|c|c|}
\hline Element & Content or meaning \\
\hline Nodes & 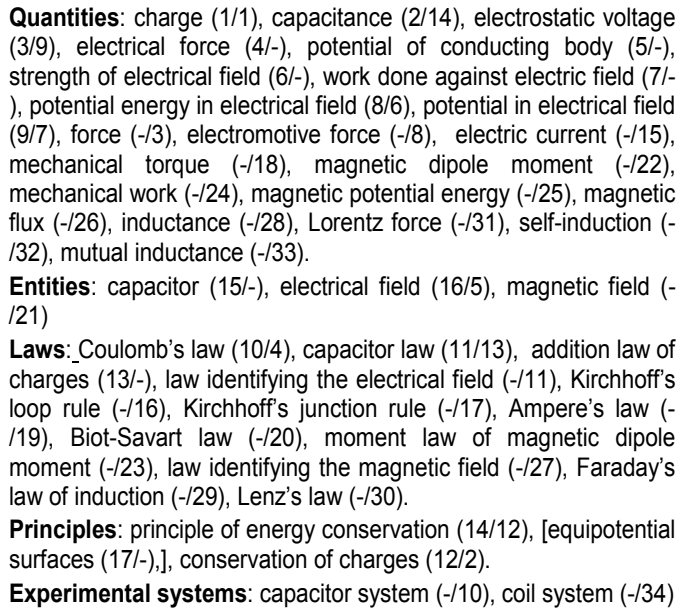 \\
\hline $\begin{array}{l}\text { Modelling } \\
\text { Procedures } \\
\text { M } \\
\text { (only part) }\end{array}$ & $\begin{array}{l}\text { M12: Electrical field of a capacitor system and transport of charge } \\
\text { (analogy to gravitational field). } \\
\text { M25: Identification of magnetic flux modelling Biot-Savart law in } \\
\text { terms of field lines. } \\
\text { M31: Modelling Ampere's law by using current element } \\
\text { interpreted in terms of the motion of charged particles. } \\
\text { M38: Modelling Faraday's induction law using field lines. }\end{array}$ \\
\hline $\begin{array}{l}\text { Experimental } \\
\text { procedures } \\
\text { E } \\
\text { (only part) }\end{array}$ & $\begin{array}{l}\text { E4: Coulomb's experiment and quantification of charge and } \\
\text { electrostatic force. } \\
\text { E21: Ampere's experiment and quantification of current and force } \\
\text { between current carrying wires. } \\
\text { E22: Biot's and Savart's experiment and quantification of field } \\
\text { strength through magnetic moment. } \\
\text { E37: Faraday's and Henry's experiment with Helmholtz's coils. } \\
\text { Quantification of induction law in terms of voltage and current. } \\
\text { Interpretation using magnetic flux. }\end{array}$ \\
\hline
\end{tabular}

\section{Concept maps in the embedded form}

The spring-embedded forms of the large $n=34$ and small $n=17$ master maps appear in Figure 4, and two examples of tree-embedded forms also appear for comparison. From the spring embeddings we can see that the concept maps in Figures 2 and 3 are well connected, web-like structures. Now the triangular patterns are also clearly visible, and their role in clustering the nodes becomes evident. When maps are drawn in tree-embedded form, the hierarchical organization of nodes becomes visible by selecting one node at a time as a root concept. The hierarchical ordering reveals the subordination of nodes to a given root node. Some nodes have a greater capacity to produce hierarchical order than do others, which makes them more central to hierarchical organisation.

The sample of 39 student maps contains very different types of concept maps, the most extensive of which have a structure comparable to that of the master maps shown in Figures 2 and 3 . Figures 5 and 6 show some examples of $n=17$ and $n=34$ student cases. 

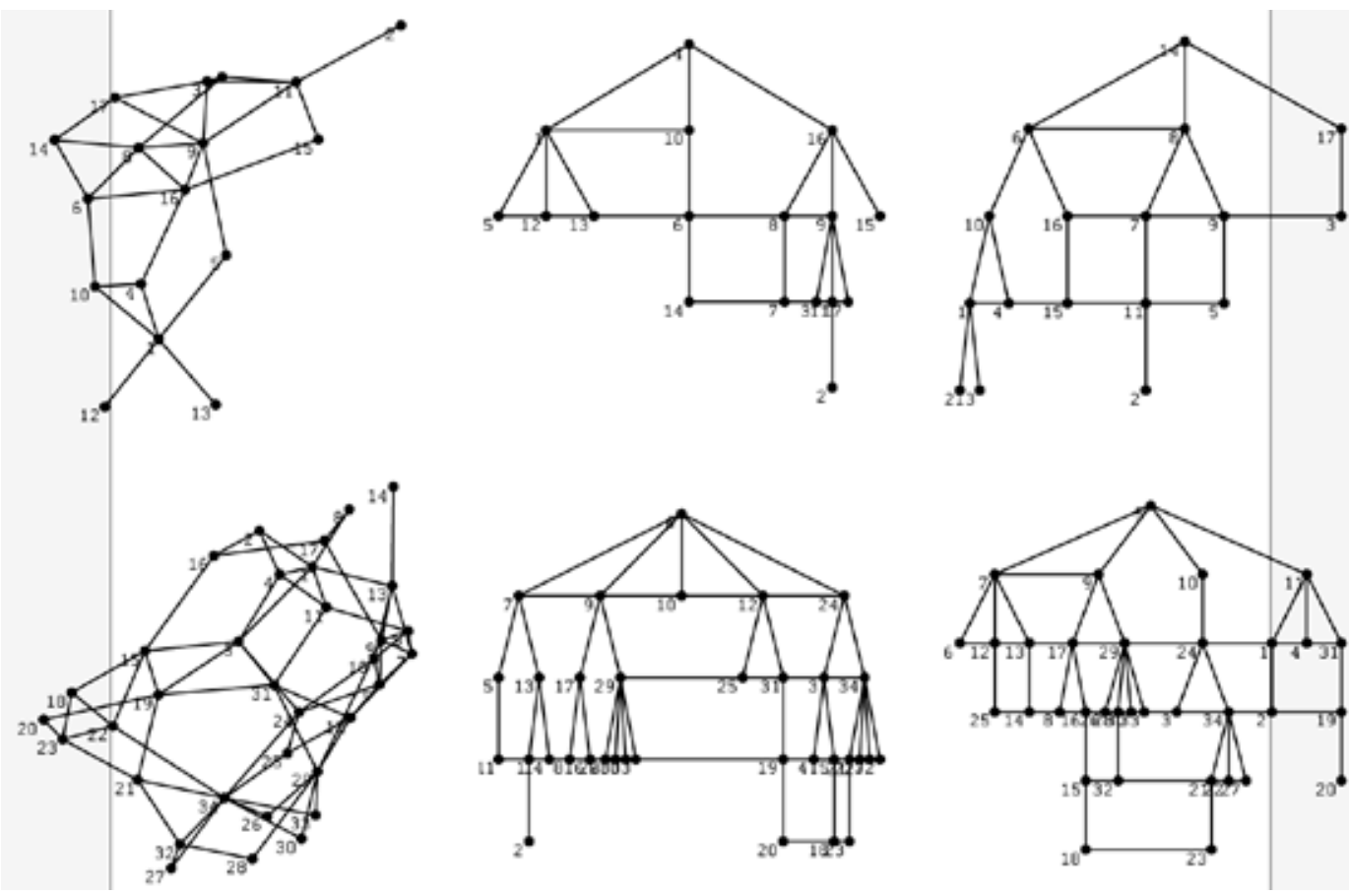

Figure 4. The concept maps (Figure 2 and 3) drawn in spring-embedded form show inbuilt hierarchies. The upper row shows the $n=17$ maps and the lower row the $n=34$ maps.
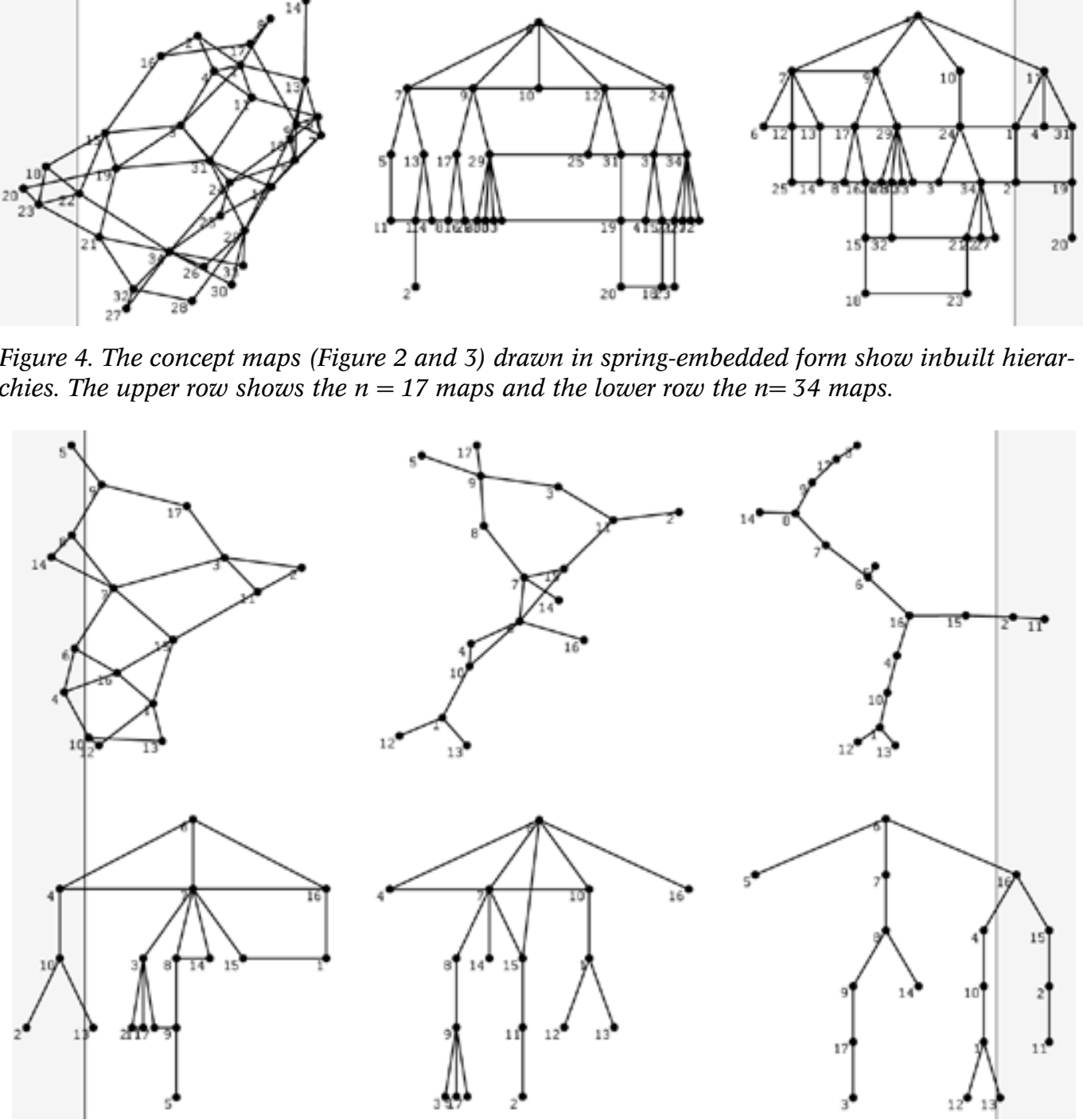

Figure 5. The students' $n=17$ concept maps. Examples showing average number of links $D=2.9$ (left), $D=2.2$ (middle) and $D=1.8$ (right). The tree-embeddings starting from node 6 (electric field strength) appear in the lower panel. 

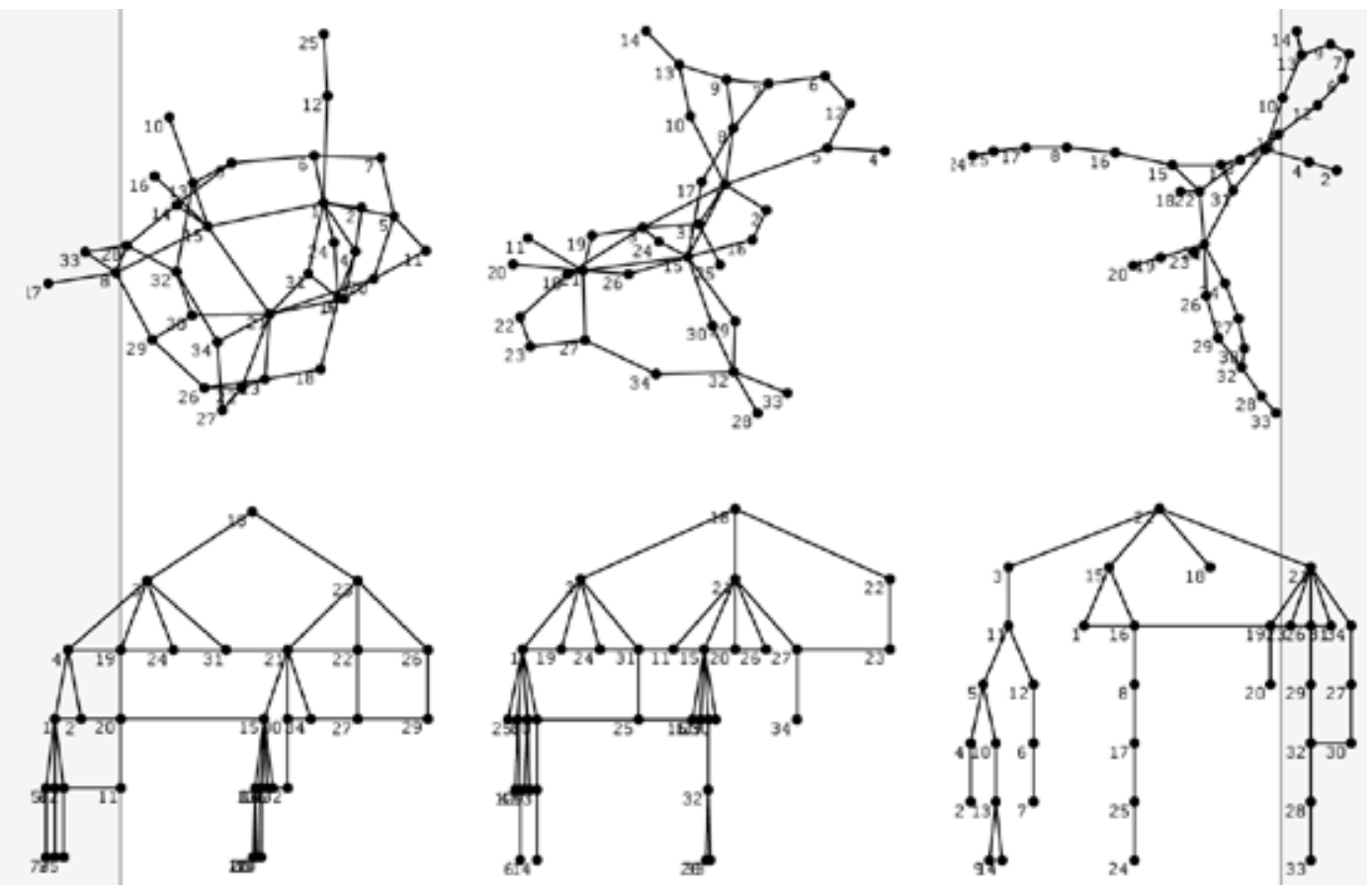

Figure 6. The students' $n=34$ concept maps. Examples showing average number of links $D=$ 3.29 (left), $D=2.71$ (middle) and $D=2.21$ (right). The tree-embeddings starting from node 18 (mechanical torque) appear in the lower panel.

The features displayed in Figures 5 and 6 are essentially those identified in recent studies of the qualitative analysis of concept maps, which emphasises the recognition of differences between web-like structures, branching structures and chain-like structures. All these qualitative notions based on visual inspection can now be operationalised and made quantitative, as the next section will show.

\section{Connecting the structure to the content}

The values of all the local variables defined in Table I were measured first from the master maps of the $n=17$ and $n=34$ concepts. Table III shows the correlations between different variables for concept maps with the $n=17$ and $n=34$ concepts. It Evidently, the correlations between the variables $C_{k}, S C_{k}$ and $I_{k}$ (the measures for clustering) are qualitatively similar, although the details depend on the size of the concept map. The results justify the notion that the importance variable $I_{k}$ measures the overall importance of the node's (concept's) structural clustering capacity. The clustering measures correlate positively with each other, but negatively with the hierarchy, which shows that $I_{k}$ and $H_{k}$ indeed measure structurally different aspects of the node.

Because $I_{k}$ and $H_{k}$ measure structurally different features, nodes with a high hierarchy are not always the concepts with the largest clustering capability or which are central to several cycles. This feature is seen particularly clearly if each node $H_{k}$ is plotted against $I_{k}$ as shown in Figure 7 . For ease of comparison, all values of small maps $n=17$ are normalised so that average values of $H_{k}$ and $I_{k}$ are the same for large and small concept maps, which permits us to compare the distribution of $H_{k}$ versus $I_{k}$ in small and large concept maps. 
Table III. Correlations (Pearson correlation coefficient) of the variables as defined in Table II and calculated for the master maps shown in Figures 3 and 4 (and in spring-and tree-embedded forms in Figure 5). The mean values appear in the first row.

\begin{tabular}{|c|c|c|c|c|c|c|}
\hline & & $D_{k}$ & $C_{k}$ & $\mathrm{SC}_{\mathrm{k}}$ & $I_{k}$ & $\mathrm{H}_{\mathrm{k}}$ \\
\hline \multirow[t]{4}{*}{$n=17$} & mean & 3.29 & 0.21 & 1.35 & 0.41 & 1.01 \\
\hline & $S C_{k}$ & 0.95 & 0.53 & & & \\
\hline & $I_{k}$ & 0.76 & 0.78 & 0.87 & & \\
\hline & $H_{k}$ & -0.64 & -0.18 & -0.70 & -0.64 & \\
\hline \multirow[t]{4}{*}{$n=34$} & mean & 4.00 & 0.20 & 1.54 & 0.41 & 1.70 \\
\hline & $S C_{k}$ & 0.96 & 0.11 & & & \\
\hline & $I_{k}$ & 0.50 & 0.68 & 0.65 & & \\
\hline & $\mathrm{H}_{\mathrm{k}}$ & -0.70 & 0.03 & -0.74 & -0.36 & \\
\hline
\end{tabular}

The data for $H_{k}$ and for $I_{k}$ in Figure 7 can be grouped into three distinct clusters by agglomerative clustering analysis (Ruskeepää, 2004). Three different distance criteria (Euclidean, Squared Euclidean and Manhattan) lead to exactly the same clustering as appears in Figure 7. The nodes are thus clustered in three distinct classes A, B and C on the basis of their roles in the topological structure of the maps. We can call these three classes clustering, hierarchy and connectivity classes.

A. The clustering class consists of nodes of high importance $\left(I_{k}>0.8\right)$ but only moderate hierarchy $\left(0.7<H_{k}<1.8\right)$ values. These nodes (concepts) are highly central, but do not necessarily produce overarching hierarchies. Concepts found in this class include: potential energy (6/8), electrical field potential (7/9), and voltage (9/3), where the first/last number refers to the map with $n=34 / n$ $=17$ respectively (see also Figures 2-3 and Table II). Clearly, these concepts are physically highly important in connecting other concepts in the maps. 


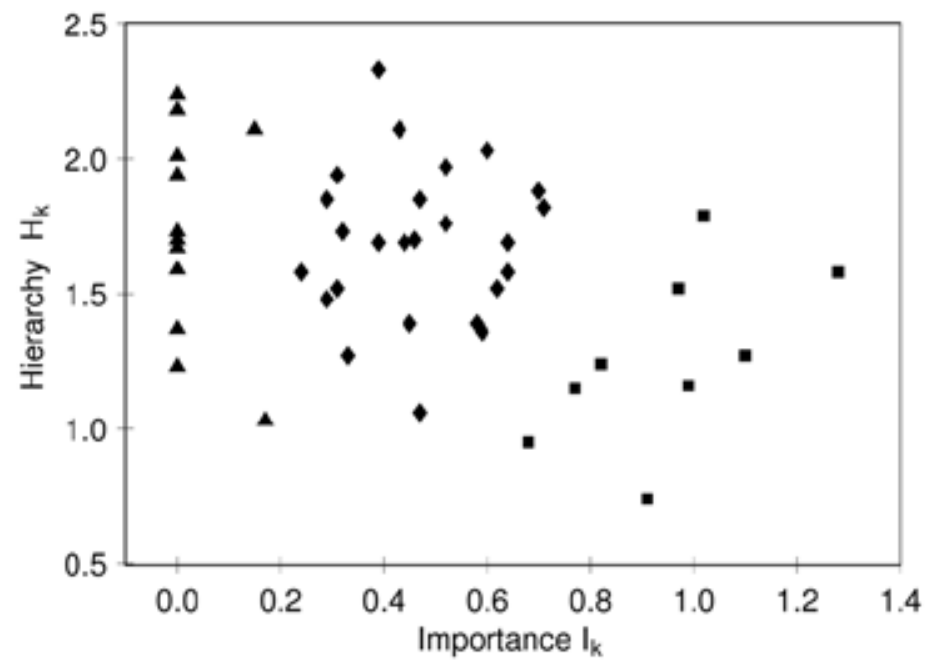

Figure. 7. Hierarchy $H_{k}$ plotted against importance $I_{k}$ for each node $k$ of the master map for $n=17$ and $n=34$. The results for $n=34$ are scaled (see text) for ease of comparisons. Different clusters found by using agglomerative clustering analysis are indicated with different symbols.

B. The hierarchy class contains nodes with $1.4<H_{k}<2.9$, but with a low clustering capacity indicated by low values $\left(I_{k}<0.2\right)$. Nodes in this class thus produce the most extensive hierarchical tree-like structures. This class contains concepts such as the addition and conservation of charges (16/12 and 13), which by nature are ordering principles or fundamental properties, as well as concepts such as capacitance (14/2) and inductance (28). These concepts are found at the far end of the chain of connections if inductively produced, or at the beginning of the chain, if used as a deductive principle.

C. The connectivity class gets its name from the notion that concepts in this class play a notable role in connecting several concepts to each other; they are equally clustering and hierarchically ordering, with $0.2<I_{k}<0.8$ and $1.0<H_{k}<2.3$. This class contains most of the concepts in the master map, central concepts such as the electrical field (16), the electrical force (4) and Coulomb's law (10) in the case of $N=17$, as well as Ampere's law (19) and Lorentz's force (31) in the case of $N=34$. These concepts play a structurally central role and are responsible for producing the overall connectivity of the structure.

The different concepts shown in the master maps thus fall into different classes, and hierarchy $H_{k}$ and importance $I_{k}$ together clearly provide a lot of information about the structure of the maps; moreover, they are also directly related to the content relevance of the concepts.

Similar information about the local structure is also available for the student concept maps considered in this study. In most cases, the concepts with an extensive hierarchy and high importance are the same as those in the case reported above. The comparison of student maps to the master maps was next carried out on the basis of the projection of the variables. The purpose of the comparison was to determine whether the concepts and laws in student maps have a similar structural position as those in the master maps. For example, we have seen that in the master maps the concepts of field and energy play central structural role, but the capacitor law is structurally less central. Figure 9 shows how the projected values for $I_{P}$ and $H_{P}$ depend on $D$. 

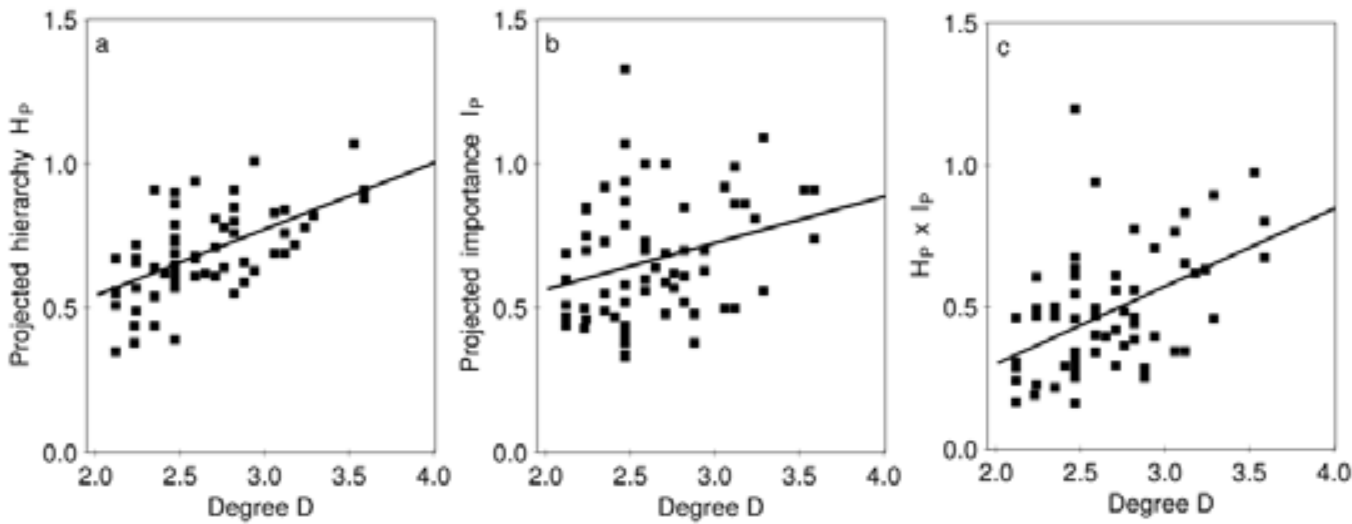

Figure 8. The projected values of hierarchy $H_{P}(a)$, importance $I_{P}(b)$ and their product $H_{P} x I_{P}(c)$ as they depend on $D$. The projected values indicate the relative difference of the students' maps from the master map (see text). The linear fit (solid line) to the data points is shown.

The result in Figures $8 \mathrm{a}$ and $8 \mathrm{~b}$ show that $I_{P}$ and $H_{P}$ are in some cases close to 1, which means that in these maps, the nodes behave very similarly to those classes $\mathrm{A}, \mathrm{B}$ and $\mathrm{C}$ discussed above, and that the structure and content are coupled in a manner similar to that in these classes. Moreover, the higher the value of $D$, the greater will be the similarity and the smaller is the variance in the data. From this we can conclude that, with increasing $D$, the content and structure in student concept maps become increasingly connected in a manner similar to that in the master maps; the concepts which are central from the point of view of subject content are indeed structurally central also. Figure $8 \mathrm{c}$ shows how the product $I_{P} \times H_{P}$ clearly increases with increasing $D$, which suggests that concept maps with good content and good structure require large $H$ and $I$. We can therefore conclude that knowledge represented in such maps is of good quality in so far as the reference point is the structure and content of the master maps.

\section{DISCUSSION AND CONCLUSIONS}

The structural validity of the analysis concerns the question of whether the variables provide information on the structural aspect we are interested in. This question is answered by noting that degree $D$ directly measures the richness of the content, whereas the structure is measured by the importance $I$ of clustering and cyclicity and by hierarchy $H$. These variables are constructed such that they operationalise the qualitative structural features of interest (see Table II).

The content validity is concerned with the question of whether the structurally important nodes are also important in regard to subject content. This is a slightly more problematic question. As Figure 8 shows, high values for $I$ and $H$ are associated with concepts (nodes) central to both structure and content at the same time. However, this conclusion rests on the analysis of the master maps and on their subsequent comparison to the student concept maps. These maps are compared by using a kind of projection of variables. Note that we have not chosen to compare them on the basis of detailed topological similarity by using, for example the adjacency matrix, which would correspond to the comparison suggested by Ruiz-Primo and Shavelson (1996) and also by McClure et al. (1999). In this case, the connections of each concept to neighbouring concepts (also occasionally called semantic connectivity) in a "master map" are compared to the connections of the same concepts in a student map. Here we have chosen a more flexible comparison on the basis of structural similarity as measured through the variables $I$ and $H$, without requiring detailed correspondence in the connections or detailed similarity of semantic fields. 
The results discussed in this study show that the most notable feature of the designed concept maps is that they have a set of nodes with a high clustering capability with respect to other nodes. Such nodes are also central to cycles in the maps (i.e. they are part of cycles) and through them map navigation is possible. It is interesting to note that this is now an acceptable conclusion for physics concepts, in particular in the context domain of electricity and magnetism. The common assumption repeatedly expressed in the literature is that concept maps in physics are expected to be hierarchical rather than connected (Novak \& Gowin, 1984; Ruiz-Primo \& Shavelson, 1996; van Zele et al., 2004). However, our results show that in addition to hierarchy, clustering, and cyclicity are equally important features of concept maps. This notion is in concordance with the recent ideas of Safayeni et al. (2005) and Derbentseva et al. (2007), who suggest that connections represented in concept maps must be more complex than simple propositions. They have also noted that, "quantification" is a necessary condition for cyclicity. These notions are also in concordance with the result that when design principles are based on procedures, which most often are quantitative experimental or modelling procedures, the concepts become interconnected and interdependent, and cyclicity becomes a consequence of this interdependence.

The research carried out here has concentrated mainly on finding the structural characteristics of concept maps and on developing methods for their analysis. Therefore, the bulk of our work is expected to impact research methodology rather than practices of using concept maps in teaching and instruction. However, the results also have implications for physics teaching and learning.

First, the results reported here suggest that concept networks can quite accurately represent organised physics knowledge and demonstrate the important role of experimental procedures and modelling procedures in the organisation of knowledge. The requirement that the design principles for making the connections be built on procedures apparently encourages students to think more carefully about the content of the connections and the consistency of the principles they are using in making the connections than about connecting the concepts simply by using propositional links. Focusing on the procedures certainly makes the students' command of the subject area visible, but also helps to foster reflective thinking during the learning process. Moreover, design principles based on procedural rules may actually help students to achieve expert-level knowledge (compare with Kharatmal \& Nagarjuna, 2008). The method for producing the conceptual networks discussed here has been in use in physics teacher education for some time and feedback from the students on its use has been much appreciated: many students have noted the advantages of being able to visualise complex conceptual connections by using the concept networks (Pehkonen et al., 2009).

Second, the results highlight the advantageous features of interconnectedness (clustering and cyclicity) because they offer greater potential to represent complex connections between concepts. Of course, several other previous studies have pointed out the importance of connectivity and cyclicity as important qualitative features of concept maps (Kinchin et al. 2000, 2005; Derbentseva et al., 2007; Safayeni et al., 2005), and here we have shown that these important structural features can coexist with hierarchical ordering.

Third, concept networks reveal interesting possibilities for monitoring conceptual development (di Sessa \& Sherin, 1998). The possibility to form well-defined quantitative measures, which characterize important structural features, provides tools for monitoring the development of expertise. The abilities of experts and advanced students are reflected in the capacity to increase the number of connections while at the same time maintaining a high degree of clustering and cyclicity in the networks. These features therefore seem to be the characteristics that signify conceptual development when expertise is gained. 
In summary, we have introduced here a quantitative method of analysis of concept maps which is capable of capturing important qualitative structural features of clustering, cyclicity and hierarchy. Such a methodological approach is needed in order to advance research focusing on concept maps that aim to understand which features of the maps may indicate beneficial learning processes. The fact that the method of analysis proposed here is necessarily more elaborate than simple visual inspection should not discourage the adoption of the method, if one aims for a better understanding of concept maps and their unambiguous classification. These results suggest that only through research, which is attentive to a rich collection of qualitative structural features but which is at the same time sufficiently deep, detailed and quantitatively accurate, can we advance our understanding of concept maps and of why they are such effective learning tools.

\section{REFERENCES}

Costa, L. da F., Rodrigues, F. A., Travieso, G., \& Villas Boas, P. R. (2007).

Characterization of complex networks: A survey of measurements. Advances in Physics, 56(1), 167-242.

Derbentseva, N., Safayeni, F., \& Cañas A. J. (2007). Concept Maps: Experiments on Dynamic Thinking. Journal of Research in Science Teaching, 44(3), 448-465.

di Sessa, A., \& Sherin, B. L. (1998). What Changes in Conceptual Change?

International Journal of Science Education, 20, 1155-1191.

Ingeç, S. K. (2009). Analysing Concept Maps as an Assessment Tool in Teaching Physics and Comparison with the Achievement Tests. International Journal of Science Education, 31(14), 1897-1915.

Kharatmal, M., \& Nagarjuna, G. (2008). Exploring roots of rigor: A proposal of a Methodology for Analyzing the Conceptual Change from a Novice to an expert, In A. J. Cañas, P. Reiska, J. D. Novak \& M. K. Åhlberg (Eds.) Concept Mapping - Connecting Educators, (pp. 391-398), Tallinn.

Kinchin, I. M., Hay, D. B., \& Adams, A. (2000). How a qualitative approach to concept map analysis can be used to aid learning by illustrating patterns of conceptual development. Educational Research, 42(1), 43-57.

Kinchin, I. M., De-Leij, F. A. A. M., \& Hay, D. B. (2005). The evolution of a collaborative concept mapping activity for undergraduate microbiology. Journal of Further and Higher Education, 29(1), 1-14.

Kolaczyk, E. D. (2009). Statistical Analysis of Network Data, New York: Springer.

Koponen, I. T., \& Pehkonen, M. (2010). Coherent Knowledge Structures of Physics Represented as Concept Networks in Teacher Education. Science \& Education,19, 259-282.

Koponen, I. T., (2007). Models and Modelling in Physics Education: A Critical Reanalysis of Philosophical Underpinnings and Suggestions for Revisions. Science \& Education, 16, 751-773.

Koponen, I. T., \& Mäntylä, T. (2006). Generative Role of Experiments in Physics and in Teaching Physics: A Suggestion for Epistemological Reconstruction Science \& Education, 15, 31-54.

McClure, J. R., Sonak, B. \& Suen, H. K. (1999). Concept Map Assessment of Classroom Learning: Reliability, Validity, and Logistical Practicality. Journal of Research in Science Teaching, 36(4), 475-492.

Nesbit, J. C., \& Adesope, O. O. (2006). Learning with Concept and Knowledge Maps: A MetaAnalysis. Review of Educational Research, 76(3) 413-448.

Novak, J., \& Gowin, B. D. (1984). Learning How to Learn. New York: Cambridge University Press.

Pehkonen, M., Koponen I. T., \& Mäntylä, T. (2009). Collaborative Concept Mapping by Using CmapTools: Supporting Conceptualisation and Learning in Higher Education, In A. Méndez Vilas, A. Solano Martín, J. Mesa González \& J. A.

Mesa González (Eds.), Researh, Reflections and Innovations in Integrating ICT in Education (pp. 347-351). Badajoz: Formatex. 
Pemmaraju, S., \& Skiena, S. (2006). Computational Discrete Mathematics: Combinatorics and Graph Theory with Mathematica. Cambridge: Cambridge University Press.

Ruiz-Primo, M. A., \& Shavelson, R. J. (1996). Problems and Issues in the Use of Concept Maps in Science Assessment. Journal of Research in Science Teaching, 33(6), 569-600. Ruskeepää, H. (2004). Mathematica Navigator: Mathematics, Statistics, and Graphs, 2nd edn. New York: Elsevier Academic Press.

Safayeni, F., Derbentseva, N., \& Cañas, A. J. (2005). A Theoretical Note on Concepts and the Need for Cyclic Concept Maps. Journal of Research in Science Teaching, 42(7), 741-766.

Sensevy, G., Tiberghien, A., Santini, J., Laube, S., \& Griggs, P. (2008). An Epistemological Approach to Modeling: Cases Studies and Implications for Science Teaching. Science Education, 92(3), 424-446.

Vanides, J., Yin, Y., Tomita, M., \& Ruiz-Primo M.A. (2005). Using concept maps in the science classroom. Science Scope, 28(8), 27-31.

Van Zele, E., Lenaerts, J., \& Wieme, W. (2004). Improving the usefulness of concept maps as a research tool for science education. International Journal of Science Education, 26(9), $1043-1064$

Zoller, U. (1990). Students' misunderstandings and misconceptions in college freshman chemistry. Journal of Research in Science Teaching, 27(10), 10531065. 